\title{
Interferon type I responses in primary and secondary infections
}

\author{
Mohammed Alsharifi, Arno Müllbacher and Matthias Regner
}

The mammalian host responds to a microbial infection with a rapid innate immune reaction that is dominated by type I interferon (IFN-I) release. Most cells of vertebrates can respond to microbial attack with IFN-I production, but the cell type responsible for most of the systemic IFN-I release is thought to be plasmacytoid dendritic cells (pDCs). Besides its antimicrobial and especially anti-viral properties IFN-I also exerts a regulatory role on many facets of the sequential adaptive immune response. One of these is being the recently described partial, systemic activation of the vast majority of $B$ and $T$ lymphocytes in mice, irrespective of antigen reactivity. The biological significance of this partial activation of lymphocytes is at present speculative. Secondary infections occurring within a short time span of a primary infection fail to elicit a similar lymphocyte activation response due to a refractory period in systemic IFN-I production. This period of exhaustion in IFN-I responses is associated with an increased susceptibility of the host to secondary infections. The latter correlates with wellestablished clinical observations of heightened susceptibility of patients to secondary microbial infections after viral episodes. Immunology and Cell Biology (2008) 86, 239-245; doi:10.1038/sj.icb.7100159; published online 8 January 2008

Keywords: type I interferon; immunoregulation; viral infections

Control of viral infections by the vertebrate host involves both the innate and the adaptive immune response. The former is mediated primarily by type I interferons (IFN-I), macrophage/monocytes and natural killer (NK) cell responses. The adaptive immune response to infection is mediated by T and B cells. Antigen-reactive T and B cells, after the primary infection has been resolved, develop the long-lasting immunological memory component. The magnitude and quality of the innate immune response are intimately involved in the subsequent adaptive primary response, which in turn determines the magnitude and quality of the memory responses. Although we have gained much insight into the mechanisms, biochemical properties and functions of the individual responses, the wider consequences of their actions are mostly still elusive. The focus of this review will be on the IFN-I responses during primary and secondary infections.

Interferon is the term given to a family of inducible glycoproteins that are produced by cells in response to biological stimuli, including viruses, bacteria, mycoplasma, protozoa, cytokines, mitogens, natural and synthetic double-stranded RNA (ds-RNA) and other substances. IFNs were discovered on the basis of their antiviral activity when influenza virus-infected chicken cells were shown to produce a secreted factor that conferred a virus-resistant state active against both homologous and heterologous viruses. ${ }^{1}$ This observation set the stage for subsequent studies that led to the elucidation of many aspects the IFN system. According to the cell types from which they were derived, three types of IFNs were originally recognized: leucocyte $(\alpha)$, fibroblast $(\beta)$ and immune $(\gamma){ }^{2,3}$ The currently accepted classification divides IFNs, based on amino-acid sequence homology, into two groups, type I (IFN-I) and type II (IFN-II). IFN-II (IFN- $\gamma$ ) is induced by mitogenic or antigenic stimuli of lymphocytes (NK and $\mathrm{T}$ cells), whereas IFN-I are induced primarily by virus infections. ${ }^{3}$ The recently identified interleukins (IL) 28 and 29 , also called IFN- $\lambda$ or IFN-III, display IFN-I-like anti-viral activities through a receptor complex distinct to that of IFN-I. ${ }^{4,5}$ Although the pattern of expression has not been elucidated yet, it is believed that different cell types, including plasmacytoid dendritic cells (pDCs), can express IFN-III during most viral infections. Their in vivo anti-viral activity, however, appears to be mediated against selected viruses. ${ }^{6,7}$

IFN-I comprise a multi-member cytokine family that includes, IFN- $\alpha$ subtypes, IFN- $\beta$, IFN- $\varepsilon$, IFN- $\kappa$, IFN- $\omega$, IFN- $\delta$ and IFN- $\tau .{ }^{8,9}$ Of these, IFN $-\delta^{10}$ and IFN- $\tau^{11}$ are not found in humans, IFN- $\varepsilon$ is expressed in the placenta and may play a role in reproduction, ${ }^{12}$ IFN- $\kappa$ is expressed in keratinocytes, ${ }^{13}$ and IFN- $\omega$ is expressed in human peripheral leucocytes following viral infection. ${ }^{14}$ From an immunological perspective, therefore, IFN- $\alpha$ and IFN- $\beta$ are the main type I subtypes of interest, and in this review we use the term IFN-I mainly to describe these two subtypes. Both are expressed in all mammalian species examined. ${ }^{2}$ In addition, they are inducible by viral infections and recognize the same multicomponent cell surface receptor, the type I IFN receptor (IFN-IR). ${ }^{15-18}$ The IFN- $\alpha$ family is a multigenic group of related polypeptides encoded by more than 13 
genes in humans and more than 10 genes in mice. ${ }^{19-21}$ IFN- $\beta$, in contrast, exists as a single gene in most species, including human and mouse. Although the reason(s) for multiple IFN- $\alpha$ subtypes is not fully understood, evidence suggests that they have distinct functions. Moreover, their expression, and the qualitative and quantitative profiles of genes they stimulate in vitro, depends on the inducing stimulus, cell type and donor. ${ }^{22}$

In addition to their important antiviral properties IFN-I exert pleiotropic effects, including inhibition of cell growth, anti-tumour activity, involvement in homoeostasis and regulatory effects on cellular and humoural immune responses. ${ }^{23}$ In this review, we briefly address IFN-I induction and antiviral activity prior to our focus on the IFN-I response in primary and secondary infections.

\section{IFN-I RESPONSE IN PRIMARY VIRAL INFECTION}

\section{Induction of IFN-I}

Generally, the production of IFN-I $(\alpha$ and $\beta$ ) is not confined to specialized cells, and most cells of vertebrates are probably capable of producing IFN-I in response to viral infection. ${ }^{23}$ Macrophages, NK cells, DCs and fibroblasts were reported to produce IFN-I in response to viral infection or exposure to microbial pathogens. ${ }^{24-28}$ However, by far the most potent producers of IFN-I are plasmacytoid DCs, which can produce up to 1000 -fold more IFN-I in vitro than any other cell type following microbial or viral challenge. ${ }^{29,30}$ These cells are thought to be responsible for the systemic IFN-I responses to many viruses. ${ }^{31,32}$ Other DCs, non-plasmacytoid, can also act as high IFN-I producer, particularly during DC-tropic viral infections. ${ }^{33}$

It is currently accepted that there are two receptor families involved in viral detection and induction of IFN-I. ${ }^{34}$ These are the Toll-like receptors (TLRs) and the cytosolic receptors (CRs). The TLR family is composed of membrane proteins expressed by different cell types, including immune cells such as macrophages, DCs, NK cells, B and T lymphocytes. ${ }^{35}$ Each TLR binds a particular type of what has been termed pathogen-associated molecular patterns, such as viral nucleic acids, lipopolysaccharides, flagellin, DNA containing cytosine phosphate guanine motifs. ${ }^{34}$ The diversity of TLRs enables the immune cells expressing them to sample the host environment for pathogen presence. In addition, their ability to recognize different types of nucleic acids allows the detection of most viral infections. Pathogen recognition by TLRs results in the transcriptional induction of multiple genes, including IFN-I. ${ }^{36,37}$ Early IFN-I themselves provide a positive autocrine feedback loop for the production of later appearing IFN types ${ }^{38}$ and a concomitant upregulation of TLRs whose ligation adds to IFN-I production, such as protein kinase R (PKR), TLR3 and TLR7. ${ }^{39,40}$

In contrast, CRs are expressed within the cytoplasm of almost all nucleated cells and thus able to recognize viral nucleic acids within the infected cells. ${ }^{34}$ Depletion studies have shown pDCs as not essential for IFN-I responses during DC-tropic lymphocytic choriomeningitis virus (LCMV) infection. ${ }^{41}$ This redundancy in pDC function is related to the ability of other non-pDC dendritic cells to act as IFN-I producer through their CRs, which recognize dsRNA. ${ }^{33}$

\section{Antiviral effects}

Although the aim of this review is not a detailed discussion of the direct antiviral effect of IFN-I, it is important to note that they were discovered on the basis of their antiviral activity. Despite the differences in the ability of cells to secrete IFN-I and of pDCs to produce large quantities, it is generally accepted that all cells can synthesize some IFN-I within hours of infection by a virus, unless protein synthesis is inhibited. This IFN-I can act in an autocrine and paracrine manner to limit replication and spread of virus. ${ }^{42}$ The early induction and action of IFN-I result in cellular resistance to viral infection, inhibition of viral replication and impediment of viral dissemination. ${ }^{43,44}$ The best-characterized direct antiviral effect by IFN-I results from the production of two ds-RNA-activated enzymes, $\left(2^{\prime}-5^{\prime}\right)$-oligoadenylate synthetase ${ }^{45}$ and ds-RNA-dependent PKR. ${ }^{46,47}$ Production of these enzymes is dependent on activation of the JAKSTAT pathway by the interaction of IFN- $\alpha / \beta$ with their specific cell surface receptor, IFN-IR. ${ }^{3,48}$ This direct effect of IFN-I results in protein synthesis inhibition in infected cells and thus limits the production of progeny viral particles. ${ }^{49}$

Many studies have shown that the direct antiviral activity of IFN-I is important in resisting many viral infections. Mice genetically deficient in functional IFN-IR (IFN-IR ${ }^{-/-}$) are extremely susceptible to viral infections such as vesicular stomatitis virus (VSV), Semliki Forest virus (SFV), vaccinia virus (VACV), LCMV,${ }^{19}$ Sindbis virus, ${ }^{50}$ Venezuelan equine encephalitis virus ${ }^{51}$ and Murray Valley encephalitis virus. $^{52}$ The role of IFN-I, however, goes beyond their ability to inhibit viral replication as they were proven to be the maestro of antiviral immunity, involved in regulating the action of almost all instruments of the immune system.

\section{Immunoregulatory role of IFN-I}

To successfully control a viral infection, virus-infected cells need to be eliminated prior to formation and release of viral progeny. Such successful outcome requires a concerted action of both the innate and adaptive arms of the immune system. IFN-I may well be the most important immunoregulators that determine the type and the level of the immune response to any particular infection. IFN-I have been reported to mediate activation of NK cell proliferation and cytotoxicity in vivo. ${ }^{53}$ However, IFN-I do not stimulate NK cell proliferation directly. They fail to elicit NK cell proliferation in vitro and exert antiproliferative effects at high concentrations, ${ }^{54}$ and their influence on NK cell responses is dependent on secondary cytokines such as IL-15. ${ }^{55,56}$ Moreover, it has been shown that, during viral infections, IFN-I-mediated activation of NK cell effector function is selective for cytotoxicity but does not extend to IFN- $\gamma$ expression. ${ }^{57}$ Furthermore, IFN-I may be crucial for antiviral responses mediated by macrophages, as they synergize with tumour necrosis factor to induce inducible nitric oxide synthase (iNOS) and iNOS-dependent pathways of defences mediated by macrophages. ${ }^{58}$

The role of IFN-I in linking innate and adaptive responses is of considerable importance. Elimination of virus-infected cells requires the generation of a virus-immune cell-mediated response which, in turn, depends on the ability of infected cells to present the viral antigens in conjunction with major histocompatibility complex (MHC) molecules. ${ }^{59}$ IFN-I induce upregulation of cell surface MHC protein expression, particularly MHC class I molecules, thereby promoting the presentation of viral antigens to $\mathrm{CD}^{+}$cytotoxic T cells. ${ }^{60}$ However, the immunoregulatory role of IFN-I goes beyond elevating the expression of MHC molecules, and may include other effects on antigen-presenting cells (APCs) as well as T and B cells.

\section{Effects on dendritic cells}

The activation of $\mathrm{T}$ and $\mathrm{B}$ lymphocytes requires the recruitment and maturation of APCs. These APCs include B cells, monocytes/macrophages and the most potent APC, DCs. While immature pDCs have been identified to be the major IFN-I producers, ${ }^{29,30}$ IFN-I themselves have been shown to play an important role in DCs maturation and survival. ${ }^{61}$ In vitro treatment of immature DCs with IFN-I induces phenotypic and functional maturation, as evident by upregulation of 
molecules involved in antigen presentation, such as MHC-I and -II, and co-stimulatory molecules, such as CD83, CD40, CD80 and CD86. ${ }^{61,62}$ Despite considerable redundancy in many cytokine functions, the effects of IFN-I on DC seem relatively unique, as only IL-15 can substitute for IFN-I in this respect, and only partially so. ${ }^{63}$ Production of IL-15 itself is induced by IFN-I, and the activating effect of IL-15 on DC is reduced in the absence of IFN-IR. ${ }^{63}$ Furthermore, in vitro treatment of DCs with IL-15 resulted in an initial production of IFN- $\beta$ followed by IFN- $\alpha{ }^{64}$

The effect of IFN-I on DC varies with the type of DC. While maturation of $\mathrm{pDCs}$ in response to TLR7/9 triggering is severely impaired in IFN-IR ${ }^{-1-}$ mice, that of conventional DC occurs normally in these mice. ${ }^{65}$ In contrast to other types of DC, in which IFN-I exposure leads to maturation into potent APC with high expression of co-stimulatory molecules, ${ }^{61,66-68}$ pDCs do not upregulate co-stimulatory molecules such as CD40, or MHC-II in response to IFN-I stimulation, ${ }^{29}$ unless directly infected by a virus. ${ }^{41}$ Not only do IFN-I promote $\mathrm{pDC}$ maturation in vitro, but their viability is also enhanced. ${ }^{29,69}$

Given the positive effect on maturation and survival of DC, it is surprising that they exert negative influence on differentiation of immature DC from bone marrow precursors. ${ }^{70}$ This inhibitory activity is such that viruses such as measles virus, LCMV and respiratory syncytial virus have developed means of immune evasion by subverting the IFN-I responses, ${ }^{70-72}$ implying that inhibition of DC precursor development provides an advantage to virus survival even at the stage when virus infection has already occurred. Thus, IFN-I mediate opposing influences on DC development, inhibiting DC precursor differentiation, but promoting the final maturation and survival of DC into initiators of the adaptive immune response.

Cytokine production by DC, in particular IL-12, has been shown to be sensitive to the abundance of IFN-I during DC activation. Low levels of IFN-I are required for IL-12p35 transcription (and consequently the production of the bioactive P70 dimer), whereas high IFN-I levels inhibit p40 transcription. ${ }^{73}$ However, complete lack of IFN-I leads to deficiency in IL-12 production.

\section{Effects on $\mathrm{T}$ and $\mathrm{B}$ lymphocytes}

The profound effects of IFN-I on DC are likely to result in significant consequences for the development of adaptive immune responses. But the pleiotropic effects of IFN-I are not confined to DC physiology, as they also directly influence $\mathrm{T}$ and $\mathrm{B}$ cells. Again, the effects may be enhancing or suppressive, depending on the circumstances. IFN-I have been shown to promote the development of effector functions of $\mathrm{T}$ cells, such as IFN- $\gamma$ production and cytolytic function, and have been termed a 'third signal' for lymphocyte activation, in addition to recognition of cognate antigen in the context of co-stimulation. ${ }^{74}$ IFN-I also promote survival of activated lymphocytes. ${ }^{75}$ Interestingly, while IFN-I-enhanced T-cell survival was shown to correspond to elevated expression of the anti-apoptotic Bcl-2 protein family member Bcl-xL in human T cells, ${ }^{76}$ mouse T cells did not display elevated levels of $\mathrm{Bcl}-\mathrm{xL}$ or Bcl-2, ${ }^{75}$ suggesting that they may be mediated by different survival mechanisms. These pro-survival effects are nevertheless likely to be caused by direct action of IFN-I on T cells, rather than via cis effects on the APCs involved in priming the $\mathrm{T}$ cells, perhaps by enhancing antigen presentation, as was showcased in cross-priming studies: IFN-I led to stronger in vivo proliferation and prolonged expansion of IFN-IR-sufficient, but not -deficient OT-I transgenic $\mathrm{T}$ cells, ${ }^{77,78}$ thereby effectively enhancing cross-priming efficiency. Again, the molecular intracellular pathways responsible for this effect remain to be characterized. Although receptors for IL-12 and -15, cytokines participating in the regulation of $\mathrm{T}$ cell survival and expansion, were upregulated, the proliferative effect of IFN-I was independent of these cytokines. Presence of IFN-I is clearly beneficial for production of IFN- $\gamma$ by antiviral CD8 T cells, ${ }^{79-81}$ and the availability of IFN-I during an antiviral T-cell response may determine whether IFN- $\gamma$ production is dependent on IL-12 or not. ${ }^{81}$

Despite this generally favourable effect of IFN-I on IFN- $\gamma$ production, early in the T-cell response the effect might be inverse. $\mathrm{T}$ cells responding to IL-12 or anti-CD3 stimulation are negatively regulated by IFN-I, at least regarding their ability to produce IFN- $\gamma{ }^{57}$ This phenomenon was also noted in $\mathrm{T}$ cells isolated in the first few days after infection with $\mathrm{LCMV}^{82}$ and, given the prominent role of IFN- $\gamma$ in activation-induced cell death (AICD) of antigen-specific T cells, this may represent a safeguard to prevent AICD of antigen-experienced $\mathrm{T}$ cells at a time when optimal expansion of such cells is still required and beneficial. However, the proliferative response of $\mathrm{T}$ cells may also be impaired by exposure in vitro to IFN-I, produced by CpGstimulated APC. ${ }^{83}$ To resolve this paradox, it was shown that this inhibition of proliferation only occurs when antigen-specific stimulation and co-stimulation are absent or suboptimal. ${ }^{84,85}$ Thus, in the absence of TCR engagement and co-stimulation, IFN-I would have a net inhibitory effect on T-cell proliferation, or may even be proapoptotic, ${ }^{86}$ whereas in their presence, IFN-I would promote optimal activation. These finely adjusted effects of IFN-I on T cells may explain the reported large scale attrition of non-virus-immune $\mathrm{CD}^{+} \mathrm{T}$ cells during early LCMV infection. ${ }^{87}$ However, it is still unclear whether T-cell attrition is a common feature to viral infection or may only be applicable to viruses that are lymphotropic. ${ }^{88,89}$ At least infection of C57BL/6 mice with the alphavirus, SFV, is not associated with T-cell loss. ${ }^{90}$ When considering these sometimes contradicting effects of IFN-I it should be noted that this can probably only be asserted for IFN-I as a whole class of molecules, and that future research may well reveal that certain activities are mediated more or less exclusively by different IFN-I subtypes. ${ }^{91}$

In addition to their effects on T cells, IFN-I regulate B cell functions such as antibody secretion and isotype switching. ${ }^{92}$ Induction of isotype switching by IFN-I may be mediated by their effect on $\mathrm{DCs}^{93}$ as well as their influence on T-cell responses. ${ }^{94}$ Co-injection of IFN-I with a soluble protein antigen enhances in vivo T-cell priming. ${ }^{62,77}$ IFN-I enhances $\mathrm{T}$ helper type 1 (Th1) responses by inhibiting the secretion of typical T helper type 2 (Th2) cytokines (IL4 and -5) but promoting production of the Th1-type cytokine IFN- $\gamma$ in $\mathrm{CD}^{+}{ }^{+} \mathrm{T}$ cells. ${ }^{94,95}$ Such effect of IFN-I is also linked to isotype switching as the increased production of IFN- $\gamma$ subsequently promotes switching of B cells to synthesise IgG $_{2 a}$ isotype antibodies. ${ }^{96}$ In addition, IFN-I promotes IgM, IgA, but not $\operatorname{IgG}_{1}$ antibody production by $\mathrm{B}$ cells. ${ }^{97}$

\section{Lymphocyte activation}

An intriguing observation is that the systemic IFN-I levels, as measured by serum levels, peak only 1 or 2 days after infection with a virus, ${ }^{26,86,98}$ with a subsequent return to baseline levels by days $3-4$, suggesting that its promoting or inhibitory activities on $\mathrm{T}$ and $\mathrm{B}$ cells are part of a programming that is imprinted at that early stage. The peak of IFN-I serum levels coincides with an almost global acquisition of a partial activation phenotype in $\mathrm{T}$ and $\mathrm{B}$ cells after viral infection, as almost all lymphocytes transiently upregulate the expression of activation markers. ${ }^{86,98}$ Given the magnitude of this phenomenon ( $>80 \%$ of all T and B cells upregulate CD69 and CD86 within a day of infection), and the relative scarcity of virus antigen-specific lymphocytes at this stage of the infection, this phenomenon is obviously 
independent of antigen reactivity. In vitro stimulation of $\mathrm{B}$ cells with IFN-I also leads to partial activation, characterized by CD69, CD86 and CD25 upregulation, but without proliferation or full differentiation into antibody-producing cells. ${ }^{99}$ Moreover, injection of poly(I:C), a synthetic ds-RNA analogue leads to proliferation of T cells in the absence of cognate antigen, albeit without upregulation of CD69 or CD25 in vivo. ${ }^{100}$ However, we recently showed that the acquisition of a partially activated phenotype by lymphocytes is dependent on IFN-I, and also appears to be a general feature of viral infection, as it was observed after infection with alphaviruses, ortho- and paramyxoviruses, adenoviruses, orthopoxviruses and flaviviruses. ${ }^{98}$ The state of partial activation observed in these cases is characterized by upregulation of CD86 and CD69 but, in contrast to the in vitro data obtained with B cells mentioned above, not CD25.

Thus, while the details vary between reports, there is agreement that IFN-I can induce a partial activation state in lymphocytes. ${ }^{98,99}$ In vitro studies have shown that treatment of B cells with recombinant IFN-I results in enhanced proliferation, receptor internalization, and signalling through the B-cell antigen receptor. ${ }^{99}$ Therefore, we suggest that induction of partial activation serves as a mechanism to transiently lower the threshold for full, antigen-dependent, activation. It is unclear, however, how IFN-I would aid in the temporary adjustment of activation thresholds. One possible mechanism is by regulating the expression of a variety of molecules, such as CD86, CD69, and CD25. The CD86 molecule is well-described co-stimulatory molecule for $\mathrm{T}$ cells when expressed on APC, but few examples have been described of CD86 expression by $\mathrm{T}$ cells themselves. In this respect, human effector memory cells have been reported to express CD86 that was able to provide co-stimulation to naive $\mathrm{T}$ cells in vitro. ${ }^{101}$ In addition, expression of functional CD86 has been demonstrated on allergenspecific $\mathrm{T}$ cells of atopic patients, ${ }^{102,103}$ where in one case CD86 was able to act in a paracrine (on naive $\mathrm{T}$ cells) but not autocrine manner, ${ }^{102}$ as well as on a small number of $\mathrm{CD}^{+}$tumour-infiltrating lymphocytes. ${ }^{104}$ However, in these instances, the $\mathrm{T}$ cells expressing CD86 were effector or memory T cells. It is conceivable, though, that CD86-CD28 ligation during $\mathrm{T}-\mathrm{T}$ interactions, for example when sampling antigen on the same APC, or when meeting in inflamed tissues, may provide stimulatory signals to both partners. Increased CD86 (as opposed to CD80) expression by DC has been suggested to be crucial for inhibition of regulatory $\mathrm{T}$ cells, thus making it an important factor in the overall effectiveness of T-cell activation. ${ }^{105-107}$ Consequently, it is tempting to speculate that T cells expressing CD86 may inhibit regulatory $\mathrm{T}$ cells particularly in situations where the suppressive function is mediated via cell-to-cell contact.

CD69, on the other hand, has long been considered an early lymphocyte activation marker. ${ }^{108}$ In fact, all bone marrow-derived cells, with the exception of erythrocytes, express CD69 upon activation. ${ }^{109}$ Given the universal association of this molecule with early activation, it is striking that specific functions of CD69, as well as a ligand, have only recently been described. While early reports proposed mainly a stimulatory contribution to lymphocyte activation, there is increasing evidence of an immunoregulatory function. ${ }^{109}$ Of particular interest is a recent finding implicating CD69 in mediating transient accumulation of lymphocytes in secondary lymphoid organs (SLO). In this report, sphingosine 1 phosphate receptor-1 (S1P1), an epithelial cell surface-expressed molecule required for lymphocyte egress from SLO, was described as a ligand for CD69. ${ }^{110}$ Thus, IFNI-induced CD69 on lymphocytes led to downregulation of S1P1, and consequent inhibition of egress of lymphocytes from, and sequestration in, SLO. ${ }^{111}$ While the main interest in these findings have been the potential of modulation of these processes for therapeutic benefit in autoimmune disease (via temporary sequestration of autoreactive $\mathrm{T}$ cells in SLO, for example during relapsing-remitting disease like multiple sclerosis), we believe that the physiological role of these processes suggest that CD69-mediated concentration of such partially activated lymphocytes in SLO may promote optimal APC-T cell interaction, and hence antigen-specific activation.

Thus, IFN-I-mediated partial lymphocyte activation may affect both, reduction of the threshold for full activation via CD86-dependent processes, while at the same time maximizing the chances of antigen-specific lymphocytes meeting their cognate antigen on APC by transiently sequestering lymphocytes in SLO during the early days of an infection.

The absence, in vivo, of another classical lymphocyte activation marker, CD25, in this generalized partial activation is likely to be important. While IFN-I may trigger a transient 'state of alert' as suggested above, CD25 is likely to only be expressed by those T cells that have, in addition to IFN-I stimulation, received further activation signals via their antigen receptors. ${ }^{78,99,112}$ In this scenario, antigen recognition may be the final, and critical, switch allowing for CD25 expression, and thereby for IL-2-dependent expansion, of antigen-specific but not bystander lymphocytes.

The manipulation of activation thresholds for lymphocytes is likely to come at a cost, though, otherwise lower thresholds would be expected to be hardwired into mature, naive lymphocytes. The evident risk is that of inadvertently breaking self-tolerance. ${ }^{113}$ It is therefore not surprising that such a state of alert is not maintained indefinitely_or expressed constitutively_but rather is temporally restricted. The expression of activation markers on lymphocytes returns to background levels within 4-5 days of infection, that is soon after the disappearance of serum IFN-I. ${ }^{98}$ We have shown that reverting lymphocytes are able to undergo renewed partial activation. ${ }^{90}$ It will be of interest to determine whether lymphocytes once 'pre-activated' by IFN-I exposure are phenotypically different from others, such as recent thymic emigrants, or those $\sim 20 \%$ of circulating cells that fail to undergo such activation during the primary infection stage, and if lymphocytes are able to undergo indefinite cycles of partial activation and reversion to a resting state.

\section{Effects on memory}

Given the multiple effects of IFN-I on the induction of adaptive immune responses, it is not surprising that they also contribute to formation and maintenance of immunological memory. $\mathrm{CD}^{+} \mathrm{T}$ cells have been shown to require a direct signal by IFN-I during initial antigen-specific activation in order to survive the subsequent contraction phase, and form memory $\mathrm{T}$ cells. ${ }^{114,115}$ In these studies, $\mathrm{CD}^{+}$ $\mathrm{T}$ cells from IFN-IR ${ }^{-1-}$ mice showed less expansion and a diminished memory pool compared to $\mathrm{CD}^{+}$cells from wild-type mice in response to infection with LCMV, VACV, VSV and Listeria monocytogenes (LM). Thus, there is a divergence in the requirements for TCR-triggering and IFN-I-mediated signals in T-cell proliferation versus survival and memory formation, respectively. Furthermore, maintenance of memory $\mathrm{T}$ cells has been suggested to be dependent on intermittent stimulation by, among other cytokines, IFN-I. In a seminal paper that rekindled interest in IFN-I functions in adaptive immunity, Tough et al demonstrated that $\mathrm{CD} 44^{\text {hi }}$ memory $\mathrm{CD} 8^{+}$cells briefly proliferated in vivo upon poly-(I:C) stimulation, before reverting to a resting phenotype. ${ }^{100}$ They also observed an increase in the pool of adoptively transferred TCR transgenic memory cells 1-2 days after poly-(I:C) injection. This suggests that IFN-I are important in maintaining immunological memory by contributing to the survival of memory $\mathrm{T}$ cells. 
In contrast, others have correlated IFN-I action with an interference with the established T-cell memory pool, in particular 'bystander' memory $\mathrm{T}$ cells. LCMV infection results in substantial lymphocyte apoptosis, which is mainly observed in the CD44hi population of memory $\mathrm{T}$ cells not reactive to LCMV-derived antigens. ${ }^{87}$ This attrition correlated with the peak of IFN-I production and was mimicked by poly-(I:C), but absent in $\mathrm{IFN}^{-\mathrm{IR}^{-1-}}$ mice. Such bystander memory cell attrition might explain the loss of T-cell memory over the course of several sequential infection with viruses unrelated to the initial infecting pathogen, ${ }^{116,117}$ and may be a necessity for generating 'space' for the expansion of antigen-specific $\mathrm{T}$ cells during an acute viral infection. Interestingly, foreign antigenreactive memory cells are relatively deficient in their response to homoeostatic signals than those $\mathrm{CD} 44^{\mathrm{hi}} \mathrm{T}$ cells that preferentially repopulate lymphopenic environments, thereby becoming diluted over time and successive infections. ${ }^{118}$

Despite widespread lymphocyte apoptosis occurring during infection with several viruses, such as LCMV and LC, Feline leukaemia virus and Feline immunodeficiency virus, ${ }^{86,87,119}$ it does not seem to be an obligatory accompaniment of viral infection, as it is not observed with alphaviruses ${ }^{120}$ or orthopoxviruses (unpublished data). Infection with LM is also characterized by significant T-cell apoptosis. ${ }^{86,121}$ The common theme here is that pathogens with tropism for lymphocytes produce this phenomenon, which suggests that fratricide may be responsible as suggested by Blanden et al 30 years ago. ${ }^{88}$

\section{IFN-I RESPONSES TO SECONDARY INFECTIONS}

As discussed above, the importance of IFN-I responses in recovery from viral infections has been clearly established. However, our information is solely based on IFN-I responses to primary infections, and little is known in regard to the induction of IFN-I during a secondary infection during or shortly after a primary infection has been resolved. It is generally anticipated that all nucleated cells have the ability to respond with IFN-I responses to a secondary viral challenge and that interferon-producing cells are able to mount such a response analogous to that seen in a primary response.

Recently, we obtained evidence in mice that the infected host undergoes a transient period of partial immune-unresponsiveness, between 5 and 9 days after an acute primary viral episode. ${ }^{120}$ This period is associated with exhausted IFN-I responses and an accompanied increased susceptibility to secondary unrelated viral infections. Indeed, we found that secreted IFN-I, the pre-requisite for systemic partial lymphocyte activation, ${ }^{98}$ was not detectable 1 day after a secondary infection when mice had previously been infected for 6 days with an unrelated virus. ${ }^{120}$ However, when secondary infections were given 9 or more days after a primary infection, IFN-I activity, as measured by lymphocyte activation status, was comparable to that seen after a primary infection. While a transient period of exhaustion in the IFN-I responses correlated with an increased susceptibility to the secondary infections, the effect of such IFN-I deficiency on regulating the immune response to the secondary infection needs to be established. Furthermore, it remains to be established why a renewed IFN-I responses is not mounted in mice at 5-9 days after a primary viral infection.

It is tempting to speculate that infected pDCs, the predominant producers of IFN-I, ${ }^{29,32}$ may be subject to elimination by cytolytic lymphocytes. Consistent with this idea is the temporal correlation of the peak of the cytotoxic T-cell response with the observed deficiency in IFN-I production. pDCs might also be depleted, irrespective of infection status, by NK cells, ${ }^{122,123}$ and may need to be replenished by homoeostatic processes. However, our observations indicate that numbers of both pDCs and conventional DCs slightly increasedrather than decreased-in spleens of aSFV-infected mice 5 days after infection. ${ }^{120}$ This is consistent with previously reported increases in pDC numbers in the spleen of herpes simplex virus (HSV)-infected mice. ${ }^{124}$ Alternatively, given the stimulus exerted by IFN-I to drive pDC maturation, ${ }^{61}$ a strong IFN-I signal might induce them to differentiate into a non-IFN-I-secreting phenotype. Previous studies have shown that DCs from HSV-infected mice fail to produce IFN-I when re-stimulated in vitro, ${ }^{124}$ and that infection of DCs with mouse cytomegalovirus resulted in their paralysis, as defined by deficiency in production of IL-12 and IL-2. ${ }^{125}$ Future investigations into these processes are likely to lead to clinically important understandings.

Clearly, the inability to respond to a viral infection with a potent IFN-I- response carries a high risk for the host, as demonstrated by the high susceptibility of IFN-I receptor-deficient mice to viral infections. ${ }^{19,50-52}$ Consistent with this, the inability to release IFN-I over a period of several days after a primary infection is associated with a striking failure to control a subsequent viral infection. ${ }^{120}$

It has been a long-standing empirical clinical observation that patients suffering from a microbial infection are often more susceptible to a secondary infection. Since IFN-I play an important antimicrobial as well as immunoregulatory role, exhaustion of the IFN-I responses may be the prime factor responsible for compromised resistance to sequential infections. Future studies aiming to address causes and consequences of IFN-I exhaustion will provide the answers and are a high priority in IFN-I research.

1 Isaacs A, Lindenmann J. Virus interference. I. The interferon. Proc R Soc Lond Biol Sci 1957; 147: 258-267.

2 Pestka S, Langer JA, Zoon KC, Samuel CE. Interferons and their actions. Annu Rev Biochem 1987; 56: 727-777.

3 Samuel CE. Antiviral actions of interferons. Clin Microbiol Rev 2001; 14: 778-809. 4 Sheppard P, Kindsvogel W, Xu W, Henderson K, Schlutsmeyer S, Whitmore TE et al. IL28, IL-29 and their class II cytokine receptor IL-28R. Nat Immunol 2003; 4: 63-68.

5 Kotenko SV, Gallagher G, Baurin VV, Lewis-Antes A, Shen M, Shah NK et al. IFNlambdas mediate antiviral protection through a distinct class II cytokine receptor complex. Nat Immunol 2003; 4: 69-77.

6 Ank N, West H, Bartholdy C, Eriksson K, Thomsen AR, Paludan SR. Lambda interferon (IFN-lambda), a type III IFN, is induced by viruses and IFNs and displays potent antiviral activity against select virus infections in vivo. J Virol 2006; 80: 4501-4509.

7 Ank N, West H, Paludan SR. IFN-lambda: novel antiviral cytokines. J Interferon Cytokine Res 2006; 26: 373-379.

8 Smith PL, Lombardi G, Foster GR. Type I interferons and the innate immune response-more than just antiviral cytokines. Mol Immunol 2005; 42: 869-877.

9 Pestka S, Krause CD, Walter MR. Interferons, interferon-like cytokines, and their receptors. Immunol Rev 2004; 202: 8-32.

10 Lefevre F, Guillomot M, D'Andrea S, Battegay S, La Bonnardiere C. Interferon-delta: the first member of a novel type I interferon family. Biochimie 1998; 80: 779-788.

11 Roberts RM, Cross JC, Leaman DW. Unique features of the trophoblast interferons. Pharmacol Ther 1991; 51: 329-345

12 Hardy MP, Owczarek CM, Jermiin LS, Ejdeback M, Hertzog PJ. Characterization of the type I interferon locus and identification of novel genes. Genomics 2004; 84: 331-345.

13 LaFleur DW, Nardelli B, Tsareva T, Mather D, Feng P, Semenuk M et al. Interferonkappa, a novel type I interferon expressed in human keratinocytes. J Biol Chem 2001; 276: 39765-39771.

14 Capon DJ, Shepard HM, Goeddel DV. Two distinct families of human and bovine interferon-alpha genes are coordinately expressed and encode functional polypeptides. Mol Cell Biol 1985; 5: 768-779.

15 Colamonici OR, Pfeffer LM, D’Alessandro F, Platanias LC, Gregory SA, Rosolen A et al. Multichain structure of the IFN-alpha receptor on hematopoietic cells. J Immunol 1992; 148: 2126-2132.

16 Novick D, Cohen B, Rubinstein M. The human interferon alpha/beta receptor: characterization and molecular cloning. Cell 1994; 77: 391-400.

17 Darnell Jr JE. STATs and gene regulation. Science 1997; 277: 1630-1635.

18 Darnell Jr JE, Kerr IM, Stark GR. Jak-STAT pathways and transcriptional activation in response to IFNs and other extracellular signaling proteins. Science 1994; 264: $1415-1421$. 
19 Muller U, Steinhoff U, Reis LF, Hemmi S, Pavlovic J, Zinkernagel RM et al. Functional role of type I and type II interferons in antiviral defense. Science 1994; 264: 1918-1921.

20 Foster GR, Finter NB. Are all type I human interferons equivalent? J Viral Hepat 1998; 5: 143-152.

21 Walter MR, Bordens R, Nagabhushan TL, Williams BR, Herberman RB, Dinarello CA et al. Review of recent developments in the molecular characterization of recombinant alfa interferons on the 40th anniversary of the discovery of interferon. Cancer Biother Radiopharm 1998; 13: 143-154.

22 Schlaak JF, Hilkens CM, Costa-Pereira AP, Strobl B, Aberger F, Frischauf AM et al. Cell-type and donor-specific transcriptional responses to interferon-alpha. Use of customized gene arrays. J Biol Chem 2002; 277: 49428-49437.

23 De Maeyer E, De Maeyer-Guignard J. Type I interferons. Int Rev Immunol 1998; 17: 53-73.

24 Belardelli F, Gresser I. The neglected role of type I interferon in the T-cell response: implications for its clinical use. Immunol Today 1996; 17: 369-372.

25 Cella M, Jarrossay D, Facchetti F, Alebardi O, Nakajima H, Lanzavecchia A et al. Plasmacytoid monocytes migrate to inflamed lymph nodes and produce large amounts of type I interferon. Nat Med 1999; 5: 919-923.

26 Eloranta ML, Sandberg K, Ricciardi-Castagnoli P, Lindahl M, Alm GV. Production of interferon-alpha/beta by murine dendritic cell lines stimulated by virus and bacteria. Scand J Immunol 1997; 46: 235-241.

27 Rodel J, Groh A, Vogelsang H, Lehmann M, Hartmann M, Straube E. Beta interferon is produced by Chlamydia trachomatis-infected fibroblast-like synoviocytes and inhibits gamma interferon-induced HLA-DR expression. Infect Immun 1998; 66: 4491-4495.

28 Sing A, Merlin T, Knopf HP, Nielsen PJ, Loppnow H, Galanos C et al. Bacterial induction of beta interferon in mice is a function of the lipopolysaccharide component. Infect Immun 2000; 68: 1600-1607.

29 Asselin-Paturel C, Boonstra A, Dalod M, Durand I, Yessaad N, Dezutter-Dambuyant C et al. Mouse type I IFN-producing cells are immature APCs with plasmacytoid morphology. Nat Immunol 2001; 2: 1144-1150.

30 Siegal FP, Kadowaki N, Shodell M, Fitzgerald-Bocarsly PA, Shah K, Ho S et al. The nature of the principal type 1 interferon-producing cells in human blood. Science 1999; 284: 1835-1837.

31 Cella M, Salio M, Sakakibara Y, Langen H, Julkunen I, Lanzavecchia A. Maturation, activation, and protection of dendritic cells induced by double-stranded RNA. J Exp Med 1999; 189: 821-829.

32 Le Bon A, Tough DF. Links between innate and adaptive immunity via type I interferon. Curr Opin Immunol 2002; 14: 432-436.

33 Diebold SS, Montoya M, Unger H, Alexopoulou L, Roy P, Haswell LE et al. Viral infection switches non-plasmacytoid dendritic cells into high interferon producers. Nature 2003; 424: 324-328.

34 Stetson DB, Medzhitov R. Type I interferons in host defense. Immunity 2006; 25: 373-381

35 Muzio M, Bosisio D, Polentarutti N, D’Amico G, Stoppacciaro A, Mancinelli R et al. Differential expression and regulation of Toll-like receptors (TLR) in human leukocytes: selective expression of TLR3 in dendritic cells. J Immunol 2000; 164: 5998-6004.

36 Uematsu S, Akira S. Toll-like Receptors and type I Interferons. J Biol Chem 2007; 282: 15319-15323.

37 Carpenter S, O'Neill L A. How important are Toll-like receptors for antimicrobial responses? Cell Microbiol 2007; 9: 1891-1901.

38 Levy DE, Marie I, Smith E, Prakash A. Enhancement and diversification of IFN induction by IRF-7-mediated positive feedback. J Interferon Cytokine Res 2002; 22: 87-93.

39 Miettinen M, Sareneva T, Julkunen I, Matikainen S. IFNs activate Toll-like receptor gene expression in viral infections. Genes Immun 2001; 2: 349-355.

40 Heinz S, Haehnel V, Karaghiosoff M, Schwarzfischer L, Muller M, Krause SW et al. Species-specific regulation of Toll-like receptor 3 genes in men and mice. J Biol Chem 2003; 278: 21502-21509.

41 Dalod M, Salazar-Mather TP, Malmgaard L, Lewis C, Asselin-Paturel C, Briere F et al. Interferon alpha/beta and interleukin 12 responses to viral infections: pathways regulating dendritic cell cytokine expression in vivo. J Exp Med 2002; 195: 517-528.

42 Deonarain R, Alcami A, Alexiou M, Dallman MJ, Gewert DR, Porter AC. Impaired antiviral response and alpha/beta interferon induction in mice lacking beta interferon. J Virol 2000; 74: 3404-3409.

43 Sen GC, Ransohoff RM. Interferon-induced antiviral actions and their regulation. Adv Virus Res 1993; 42: 57-102.

44 van den Broek MF, Muller U, Huang S, Zinkernagel RM, Aguet M. Immune defence in mice lacking type I and/or type II interferon receptors. Immunol Rev 1995; 148: 5-18.

45 Zhou A, Paranjape J, Brown TL, Nie H, Naik S, Dong B et al. Interferon action and apoptosis are defective in mice devoid of $2^{\prime}, 5^{\prime}$-oligoadenylate-dependent RNase L. EMBO J 1997; 16: 6355-6363.

46 Leib DA, Machalek MA, Williams BR, Silverman RH, Virgin HW. Specific phenotypic restoration of an attenuated virus by knockout of a host resistance gene. Proc Nat Acad Sci USA 2000; 97: 6097-6101.

47 Bergmann M, Garcia-Sastre A, Carnero E, Pehamberger H, Wolff K, Palese P et al. Influenza virus NS1 protein counteracts PKR-mediated inhibition of replication. J Virol 2000; 74: 6203-6206.

48 Levy DE, Garcia-Sastre A. The virus battles: IFN induction of the antiviral state and mechanisms of viral evasion. Cytokine Growth Factor Rev 2001; 12: 143-156.
49 Jaramillo ML, Abraham N, Bell JC. The interferon system: a review with emphasis on the role of PKR in growth control. Cancer Invest 1995; 13: 327-338.

50 Ryman KD, Klimstra WB, Nguyen KB, Biron CA, Johnston RE. Alpha/beta interferon protects adult mice from fatal Sindbis virus infection and is an important determinant of cell and tissue tropism. J Virol 2000; 74: 3366-3378.

51 White LJ, Wang JG, Davis NL, Johnston RE, Role of alpha/beta interferon in Venezuelan equine encephalitis virus pathogenesis: effect of an attenuating mutation in the $5^{\prime}$ untranslated region. J Virol 2001; 75: 3706-3718.

52 Lobigs M, Mullbacher A, Wang Y, Pavy M, Lee E. Role of type I and type II interferon responses in recovery from infection with an encephalitic flavivirus. J Gen Virol 2003; 84: 567-572.

53 Biron CA, Nguyen KB, Pien GC, Cousens LP, Salazar-Mather TP. Natural killer cells in antiviral defense: function and regulation by innate cytokines. Annu Rev Immunol 1999; 17: 189-220.

54 Jewett A, Bonavida B. Interferon-alpha activates cytotoxic function but inhibits interleukin-2-mediated proliferation and tumor necrosis factor-alpha secretion by immature human natural killer cells. J Clin Immunol 1995; 15: 35-44.

55 Becknell B, Caligiuri MA. Interleukin-2, interleukin-15, and their roles in human natural killer cells. Adv Immunol 2005; 86: 209-239.

56 Waldmann TA, Tagaya Y. The multifaceted regulation of interleukin- 15 expression and the role of this cytokine in NK cell differentiation and host response to intracellular pathogens. Annu Rev Immunol 1999; 17: 19-49.

57 Nguyen KB, Cousens LP, Doughty LA, Pien GC, Durbin JE, Biron CA. Interferon alpha/ beta-mediated inhibition and promotion of interferon gamma: STAT1 resolves a paradox. Nat Immunol 2000; 1: 70-76.

58 MacMicking J, Xie QW, Nathan C. Nitric oxide and macrophage function. Annu Rev Immunol 1997; 15: 323-350.

59 Zinkernagel RM, Doherty PC. Restriction of in vitro T cell-mediated cytotoxicity in lymphocytic choriomeningitis within a syngeneic or semiallogeneic system. Nature 1974; 248: 701-702.

60 Boehm U, Klamp T, Groot M, Howard JC. Cellular responses to interferon-gamma. Annu Rev Immunol 1997; 15: 749-795.

61 Luft T, Pang KC, Thomas E, Hertzog P, Hart DN, Trapani J et al. Type I IFNs enhance the terminal differentiation of dendritic cells. J Immunol 1998; 161: 1947-1953.

62 Gallucci S, Lolkema M, Matzinger P. Natural adjuvants: endogenous activators of dendritic cells. Nat Med 1999; 5: 1249-1255.

63 Mattei F, Schiavoni G, Belardelli F, Tough DF. IL-15 is expressed by dendritic cells in response to type I IFN, double-stranded RNA, or lipopolysaccharide and promotes dendritic cell activation. J Immunol 2001; 167: 1179-1187.

64 Jinushi M, Takehara T, Tatsumi T, Kanto T, Groh V, Spies T et al. Autocrine/paracrine IL-15 that is required for type I IFN-mediated dendritic cell expression of $\mathrm{MHC}$ class Irelated chain A and B is impaired in hepatitis C virus infection. J Immunol 2003; 171: 5423-5429.

65 Asselin-Paturel C, Brizard G, Chemin K, Boonstra A, O'Garra A, Vicari A et al. Type I interferon dependence of plasmacytoid dendritic cell activation and migration. $J$ Exp Med 2005; 201: 1157-1167.

66 Paquette RL, Hsu N, Said J, Mohammed M, Rao NP, Shih G et al. Interferon-alpha induces dendritic cell differentiation of CML mononuclear cells in vitro and in vivo. Leukemia 2002; 16: 1484-1489.

67 Santodonato L, D'Agostino G, Nisini R, Mariotti S, Monque DM, Spada M et al. Monocyte-derived dendritic cells generated after a short-term culture with IFN-alpha and granulocyte-macrophage colony-stimulating factor stimulate a potent EpsteinBarr virus-specific CD8+ T cell response. J Immunol 2003; 170: 5195-5202.

68 Honda K, Sakaguchi S, Nakajima C, Watanabe A, Yanai H, Matsumoto M et al. Selective contribution of IFN-alpha/beta signaling to the maturation of dendritic cells induced by double-stranded RNA or viral infection. Proc Natl Acad Sci USA 2003; 100: 10872-10877.

69 Kadowaki N, Antonenko S, Lau JY, Liu YJ. Natural interferon alpha/beta-producing cells link innate and adaptive immunity. J Exp Med 2000; 192: 219-226.

70 Hahm B, Trifilo MJ, Zuniga El, Oldstone MB. Viruses evade the immune system through type I interferon-mediated STAT2-dependent, but STAT1-independent, signaling. Immunity 2005; 22: 247-257.

71 Schlender J, Hornung V, Finke S, Gunthner-Biller M, Marozin S, Brzozka K et al. Inhibition of Toll-like receptor 7- and 9-mediated alpha/beta interferon production in human plasmacytoid dendritic cells by respiratory syncytial virus and measles virus. J Virol 2005; 79: 5507-5515.

72 Ramaswamy M, Shi L, Monick MM, Hunninghake GW, Look DC. Specific inhibition of type I interferon signal transduction by respiratory syncytial virus. Am J Respir Cell Mol Biol 2004; 30: 893-900.

73 Gautier G, Humbert M, Deauvieau F, Scuiller M, Hiscott J, Bates EE et al. A type I interferon autocrine-paracrine loop is involved in Toll-like receptor-induced interleukin-12p70 secretion by dendritic cells. J Exp Med 2005; 201: 1435-1446.

74 Curtsinger JM, Lins DC, Mescher MF. Signal 3 determines tolerance versus full activation of naive CD8T cells: dissociating proliferation and development of effector function. J Exp Med 2003; 197: 1141-1151.

75 Marrack P, Kappler J, Mitchell T. Type I interferons keep activated T cells alive. J Exp Med 1999; 189: 521-530.

76 Pilling D, Akbar AN, Girdlestone J, Orteu CH, Borthwick NJ, Amft N et al. Interferonbeta mediates stromal cell rescue of T cells from apoptosis. Eur J Immunol 1999; 29: 1041-1050.

77 Le Bon A, Etchart N, Rossmann C, Ashton M, Hou S, Gewert D et al. Cross-priming of $\mathrm{CD}^{+} \mathrm{T}$ cells stimulated by virus-induced type I interferon. Nat Immunol 2003; 4: 1009-1015. 
78 Le Bon A, Durand V, Kamphuis E, Thompson C, Bulfone-Paus S, Rossmann C et al. Direct stimulation of $\mathrm{T}$ cells by type I IFN enhances the $\mathrm{CD} 8^{+} \mathrm{T}$ cell response during cross-priming. J Immunol 2006; 176: 4682-4689.

79 Nguyen KB, Watford WT, Salomon R, Hofmann SR, Pien GC, Morinobu A et al. Critical role for STAT4 activation by type 1 interferons in the interferon-gamma response to viral infection. Science 2002; 297: 2063-2066.

80 Pien GC, Nguyen KB, Malmgaard L, Satoskar AR, Biron CA. A unique mechanism for innate cytokine promotion of T cell responses to viral infections. J Immunol 2002; 169: 5827-5837.

81 Cousens LP, Peterson R, Hsu S, Dorner A, Altman JD, Ahmed R et al. Two roads diverged: interferon alpha/beta- and interleukin 12-mediated pathways in promoting $T$ cell interferon gamma responses during viral infection. J Exp Med 1999; 189: 1315-1328.

82 Liu F, Whitton JL. Cutting edge: re-evaluating the in vivo cytokine responses of $\mathrm{CD}^{+} \mathrm{T}$ cells during primary and secondary viral infections. J Immunol 2005; 174: 59365940.

83 Sun S, Zhang $X$, Tough DF, Sprent J. Type I interferon-mediated stimulation of T cells by CpG DNA. J Exp Med 1998; 188: 2335-2342.

84 Sun S, Zhang X, Tough D, Sprent J. Multiple effects of immunostimulatory DNA on T cells and the role of type I interferons. Springer Semin Immunopathol 2000; 22: 77-84.

85 Sprent J, Zhang X, Sun S, Tough D. T-cell proliferation in vivo and the role of cytokines. Philos Trans R Soc Lond B Biol Sci 2000; 355: 317-322.

86 Jiang J, Lau LL, Shen H. Selective depletion of nonspecific T cells during the early stage of immune responses to infection. J Immunol 2003; 171: 4352-4358.

87 McNally JM, Zarozinski CC, Lin MY, Brehm MA, Chen HD, Welsh RM. Attrition of bystander CD8 T cells during virus-induced T-cell and interferon responses. J Virol 2001; 75: 5965-5976.

88 Dunlop MB, Blanden RV. Mechanisms of suppression of cytotoxic T-cell responses in murine lymphocytic choriomeningitis virus infection. J Exp Med 1977; 145: 1131-1143.

89 Hanon E, Stinchcombe JC, Saito M, Asquith BE, Taylor GP, Tanaka Y et al. Fratricide among CD8(+) T lymphocytes naturally infected with human T cell lymphotropic virus type I. Immunity 2000; 13: 657-664.

90 Alsharifi M, Lobigs M, Simon MM, Kersten A, Muller K, Koskinen A et al. NK cell-mediated immunopathology during an acute viral infection of the CNS. Eur $J$ Immunol 2006; 36: 887-896.

91 Bogdan C, Mattner J, Schleicher U. The role of type I interferons in non-viral infections. Immunol Rev 2004; 202: 33-48.

92 Stark GR, Kerr IM, Williams BR, Silverman RH, Schreiber RD. How cells respond to interferons. Annu Rev Biochem 1998; 67: 227-264.

93 Le Bon A, Schiavoni G, D'Agostino G, Gresser I, Belardelli F, Tough DF. Type I interferons potently enhance humoral immunity and can promote isotype switching by stimulating dendritic cells in vivo. Immunity 2001; 14: 461-470.

94 Demeure $\mathrm{CE}, \mathrm{Wu} \mathrm{CY}$, Shu U, Schneider PV, Heusser $\mathrm{C}$, Yssel $\mathrm{H}$ et al. In vitro maturation of human neonatal CD4 T lymphocytes. II. Cytokines present at priming modulate the development of lymphokine production. J Immunol 1994; 152: 4775-4782.

95 Brinkmann V, Geiger T, Alkan S, Heusser $\mathrm{CH}$. Interferon alpha increases the frequency of interferon gamma-producing human $\mathrm{CD}^{+} \mathrm{T}$ cells. J Exp Med 1993; 178: 1655-1663.

96 Sinigaglia F, D'Ambrosio D, Rogge L. Type I interferons and the Th1/Th2 paradigm. Dev Comp Immunol 1999; 23: 657-663.

97 Estes DM, Tuo W, Brown WC, Goin J. Effects of type I/type II interferons and transforming growth factor-beta on B-cell differentiation and proliferation. Definition of costimulation and cytokine requirements for immunoglobulin synthesis and expression. Immunology 1998; 95: 604-611.

98 Alsharifi M, Lobigs M, Regner M, Lee E, Koskinen A, Mullbacher A. Type-I interferons, in response to viral infection, trigger systemic, partial, lymphocyte activation. J Immunol 2005; 175: 4635-4640.

99 Braun D, Caramalho I, Demengeot J. IFN-alpha/beta enhances BCR-dependent B cell responses. Int Immunol 2002; 14: 411-419.

100 Tough DF, Borrow P, Sprent J. Induction of bystander T cell proliferation by viruses and type I interferon in vivo. Science 1996; 272: 1947-1950.

101 Jeannin P, Herbault N, Delneste Y, Magistrelli G, Lecoanet-Henchoz S, Caron G et al. Human effector memory $T$ cells express CD86: a functional role in naive $T$ cell priming. J Immunol 1999; 162: 2044-2048.

102 Nakada M, Nishizaki K, Yoshino T, Okano M, Yamamoto T, Masuda Y et al. CD80 (B71 ) and CD86 (B7-2) antigens on house dust mite-specific T cells in atopic disease function through T-T cell interactions. J Allergy Clin Immunol 1999; 104: 222-227.
103 Hattori H, Okano M, Yoshino T, Akagi T, Nakayama E, Saito C et al. Expression of costimulatory CD80/CD86-CD28/CD152 molecules in nasal mucosa of patients with perennial allergic rhinitis. Clin Exp Allergy 2001; 31: 1242-1249.

104 Melichar B, Nash MA, Lenzi R, Platsoucas CD, Freedman RS. Expression of costimulatory molecules CD80 and CD86 and their receptors CD28, CTLA-4 on malignant ascites $\mathrm{CD}^{+}$tumour-infiltrating lymphocytes (TIL) from patients with ovarian and other types of peritoneal carcinomatosis. Clin Exp Immunol 2000; 119: 19-27.

105 Manzotti CN, Tipping H, Perry LC, Mead KI, Blair PJ, Zheng Y et al. Inhibition of human $T$ cell proliferation by CTLA-4 utilizes CD80 and requires $C D 25^{+}$regulatory T cells. Eur J Immunol 2002; 32: 2888-2896.

106 Takahashi T, Hirano N, Takahashi T, Chiba S, Yazaki Y, Hirai H. Immunogene therapy against mouse leukemia using B7 molecules. Cancer Gene Ther 2000; 7: 144-150.

107 Zheng Y, Manzotti CN, Liu M, Burke F, Mead KI, Sansom DM. CD86 and CD80 differentially modulate the suppressive function of human regulatory $T$ cells. J Immunol 2004; 172: 2778-2784.

108 Testi R, D'Ambrosio D, De Maria R, Santoni A. The CD69 receptor: a multipurpose cell-surface trigger for hematopoietic cells. Immunol Today 1994; 15: 479-483.

109 Sancho D, Gomez M, Sanchez-Madrid F. CD69 is an immunoregulatory molecule induced following activation. Trends Immunol 2005; 26: 136-140.

110 Matloubian M, Lo CG, Cinamon G, Lesneski MJ, Xu Y, Brinkmann V et al. Lymphocyte egress from thymus and peripheral lymphoid organs is dependent on S1P receptor 1. Nature 2004; 427: 355-360.

111 Shiow LR, Rosen DB, Brdickova N, Xu Y, An J, Lanier LL et al. CD69 acts downstream of interferon-alpha/beta to inhibit S1P1 and lymphocyte egress from lymphoid organs. Nature 2006; 440: 540-544.

112 Lowenthal JW, Tougne C, MacDonald HR, Smith KA, Nabholz M. Antigenic stimulation regulates the expression of IL 2 receptors in a cytolytic T lymphocyte clone. $\mathrm{J}$ Immunol 1985; 134: 931-939.

113 Baechler EC, Gregersen PK, Behrens TW. The emerging role of interferon in human systemic lupus erythematosus. Curr Opin Immunol 2004; 16: 801-807.

114 Kolumam GA, Thomas S, Thompson LJ, Sprent J, Murali-Krishna K. Type I interferons act directly on CD8 $T$ cells to allow clonal expansion and memory formation in response to viral infection. J Exp Med 2005; 202: 637-650.

115 Thompson LJ, Kolumam GA, Thomas S, Murali-Krishna K. Innate inflammatory signals induced by various pathogens differentially dictate the IFN-I dependence of CD8 T cells for clonal expansion and memory formation. J Immunol 2006; 177: 1746-1754.

116 Selin LK, Vergilis K, Welsh RM, Nahill SR. Reduction of otherwise remarkably stable virus-specific cytotoxic $\mathrm{T}$ lymphocyte memory by heterologous viral infections. J Exp Med 1996; 183: 2489-2499.

117 Selin LK, Lin MY, Kraemer KA, Pardoll DM, Schneck JP, Varga SM et al. Attrition of T cell memory: selective loss of LCMV epitope-specific memory CD8 T cells following infections with heterologous viruses. Immunity 1999; 11: 733-742.

118 Peacock CD, Kim SK, Welsh RM. Attrition of virus-specific memory $\mathrm{CD}^{+}$ T cells during reconstitution of lymphopenic environments. J Immunol 2003; 171: 655-663.

119 Tompkins MB, Nelson PD, English RV, Novotney C. Early events in the immunopathogenesis of feline retrovirus infections. J Am Vet Med Assoc 1991; 199: 1311-1315.

120 Alsharifi M, Regner M, Blanden R, Lobigs M, Lee E, Koskinen A et al. Exhaustion of type I interferon response following an acute viral infection. J Immunol 2006; 177: 3235-3241.

121 Carrero JA, Calderon B, Unanue ER. Listeriolysin O from Listeria monocytogenes is a lymphocyte apoptogenic molecule. J Immunol 2004; 172: 4866-4874.

122 Hayakawa Y, Screpanti V, Yagita H, Grandien A, Ljunggren HG, Smyth MJ et al. NK cell TRAIL eliminates immature dendritic cells in vivo and limits dendritic cell vaccination efficacy. J Immunol 2004; 172: 123-129.

123 Della Chiesa M, Vitale M, Carlomagno S, Ferlazzo G, Moretta L, Moretta A. The natural killer cell-mediated killing of autologous dendritic cells is confined to a cell subset expressing CD94/NKG2A, but lacking inhibitory killer lg-like receptors. Eur J Immunol 2003; 33: 1657-1666.

124 Bjorck P. Dendritic cells exposed to herpes simplex virus in vivo do not produce IFNalpha after rechallenge with virus in vitro and exhibit decreased T cell alloreactivity. $\mathrm{J}$ Immunol 2004; 172: 5396-5404.

125 Andrews DM, Andoniou CE, Granucci F, Ricciardi-Castagnoli P, Degli-Esposti MA. Infection of dendritic cells by murine cytomegalovirus induces functional paralysis. Nat Immunol 2001; 2: 1077-1084. 